\title{
Current status and future perspectives of PSMA-targeted therapy in Europe: opportunity knocks
}

\author{
A. Pfestroff ${ }^{1}$ - M. Luster ${ }^{1}$ - C. A. Jilg $^{2}$ - P. J. Olbert ${ }^{3}$ - C. H. Ohlmann ${ }^{4}$ - M. Lassmann ${ }^{5}$. \\ H. R. Maecke ${ }^{6} \cdot$ S. Ezziddin $^{7} \cdot$ L. Bodei $^{8} \cdot$ on behalf of the Radionuclide Therapy \\ Committee of the European Association of Nuclear Medicine
}

Published online: 16 September 2015

(C) Springer-Verlag Berlin Heidelberg 2015

Prostate cancer (PCA), the second most common cancer in men and the fourth most common malignancy overall, causes an estimated 90,000 deaths per year in Europe [1]. Castrationresistant PCA (CRPC) is defined according to the Prostate Cancer Working Group 2 criteria as PCA with any progression occurring in the presence of castrate-level testosterone values. This progression may be biochemical, i.e. a rise in prostatespecific antigen (PSA) levels, or clinical, i.e. appearance of metastases on imaging [2].

The CRPC therapeutic landscape has changed dramatically over the last decade. In 2003, the only options for patients

M. Luster

luster@med.uni-marburg.de

1 Department of Nuclear Medicine, University Hospital Marburg, Marburg, Germany

2 Department of Urology, University Hospital Freiburg, Freiburg, Germany

3 Department of Urology, University Hospital Marburg, Marburg, Germany

4 Department of Urology, Saarland University Hospital, Homburg/Saar, Germany

5 Department of Nuclear Medicine, University Hospital Würzburg, Würzburg, Germany

6 Department of Nuclear Medicine and Radiopharmacy, University Hospital Freiburg, Freiburg, Germany

7 Department of Nuclear Medicine, Saarland University Hospital, Homburg/Saar, Germany

8 Department of Nuclear Medicine, European Institute of Oncology, Milan, Italy when medical or surgical castration and peripheral androgen blockade had failed were palliative chemotherapy with mitoxantrone or best supportive care, including symptomatic palliative radiation or corticosteroids. As of 2015, five compounds have been approved for treating CRPC. Each has been demonstrated in pivotal phase III trials to confer an overall survival benefit (Table 1) [3-10].

Docetaxel was the mainstay of therapy for several years, before abiraterone/prednisolone, enzalutamide, cabazitaxel and the alpha-emitter ${ }^{223} \mathrm{Ra}$ entered the stage. Every one of these substances not only improves survival endpoints but also provides numerous palliative benefits, e.g. pain control, quality-of-life improvement, and prevention of skeletal events [3-10]. The optimal sequence of this variety of options, however, especially the optimal positioning of chemotherapy in relation to hormonal manipulation or ${ }^{223} \mathrm{Ra}$ administration, remains unclear, as does the potential of combinations of compounds. Trials to investigate these questions are underway. Despite these advances, overall survival for patients with CRPC remains relatively short, e.g., a median 19 months for patients in 23 Phase 3 trials of novel therapies $(n=13909)$ [11]. Thus the search for CRPC treatments continues.

The implementation of radiolabelled compounds targeting prostate-specific membrane antigen (PSMA) for both diagnostic and therapeutic applications is considered to be a milestone in the management of these patients. PSMA PET/CT offers an appealing combination of PCA specificity and high sensitivity at low tumour volumes. These characteristics have led to the evolution of PSMA PET/CT into an important diagnostic tool in the management of advanced PCA. In the course of this evolution, it has become apparent that PSMA expression persists in a high percentage of patients with CRPC - in contrast to the expression of 
Table 1 Compounds approved for the treatment of CRPC: overall survival benefits versus control arms in phase III clinical studies

\begin{tabular}{|c|c|c|c|c|c|}
\hline Reference & Investigational compound & Control arm & $\begin{array}{l}\text { Hazard ratio for } \\
\text { death }(95 \% \mathrm{CI})\end{array}$ & $\begin{array}{l}\text { Overall survival } \\
\text { benefit (months) }\end{array}$ & $P$ value \\
\hline [3] & Docetaxel & Mitoxantrone & $0.80(0.67-0.94)$ & 1.9 & 0.02 \\
\hline [4] & Docetaxel & Mitoxantrone & $0.76(0.64-0.94)$ & 2.4 & 0.009 \\
\hline [5] & Cabazitaxel after docetaxel & Mitoxantrone & $0.70(0.59-0.83)$ & 2.1 & 0.001 \\
\hline [6] & Abiraterone after docetaxel & Placebo & $0.65(0.54-0.77)$ & 3.9 & 0.001 \\
\hline [7] & Abiraterone before docetaxel & Placebo & $0.75(0.61-0.93)$ & 5.2 & 0.0097 \\
\hline [8] & Enzalutamide after docetaxel & Placebo & $0.63(0.53-0.75)$ & 4.8 & 0.001 \\
\hline [9] & Enzalutamide before docetaxel & Placebo & $0.71(0.60-0.84)$ & 1.8 & 0.001 \\
\hline [10] & ${ }^{223} \mathrm{Ra}$ & Placebo & $0.70(0.56-0.83)$ & 3.6 & 0.00007 \\
\hline
\end{tabular}

blood biomarkers such as PSA. Thus PSMA represents an intriguing "theragnostic" target in the CRPC setting $[12,13]$.

The observation of frequent persistent PSMA expression in patients with CRPC has provided the rationale for the recent introduction of PSMA radioligand therapy, with promising initial results $[14,15]$. To develop this modality further, it is now necessary for nuclear medicine physicians and urologists to cooperate closely, to set up standards for the best conduct of PSMA-based radiotherapy, and to initiate prospective clinical trials. These studies should have carefully considered objectives and well-defined endpoints, and thus involve comparable patient cohorts. Such actions could enable PSMA-directed radionuclide therapy to find its way to regulatory approval and into clinical practice.

\section{The target and radiolabelled peptidomimetic ligands for "theragnostic" applications}

An ideal molecular target (biomarker) for oncological imaging and radionuclide therapy should be specific (potentially unique to the tumour), easily accessible at the tumour cell plasma membrane, biologically relevant, highly expressed, and not shed into the circulation. PSMA, a 750-amino acid type II transmembrane glycoprotein, appears to largely fulfil these requirements. PSMA is upregulated in PCA and on the neovasculature of several other human solid malignancies. The glycoprotein's expression is low on normal prostate tissue, but elevated in PCA. PSMA is also expressed to some extent in the salivary and lacrimal glands, in the small intestine, and particularly in the kidneys, although this expression is markedly less than that on prostate tumour. The expression of PSMA seems to be upregulated in advanced disease. This characteristic presents a yet-unexplored opportunity in targeted radionuclide therapy since the majority of malignancies are generally considered to lose their specific markers, i.e. potential targets, in the course of the disease.

Initially, monoclonal antibodies were raised against PSMA, and radiolabelled, and these compounds were evaluated in clinical trials [16]. Recently, small urea-based molecules have turned out to be the preferred family of PSMA-targeting compounds. The pharmacophoric component of these molecules is the Glu-urea-Lys unit [17]. For imaging, a range of smallmolecule PSMA-targeting ligands with a variety of radionuclides have been studied preclinically and clinically for SPECT, PET and radioguided surgery $[18,19]$. Among them, ${ }^{68} \mathrm{Ga}$-PSMA-HBED-CC appears to be the current clinical gold standard [20]. For targeted radionuclide therapy, two peptidomimetics have been developed. One of these is Glu-NH-CO-NH-Lys-spacer-1,4,7,10-tetraazacyclododecane1-(glutaric acid)-4,7,10-triacetic acid (DOTAGA). The spacer is composed of lysine aliphatic chains as well as aromatic amino acids such as phenylalanine. A subsequently developed version of this peptidomimetic used a D-amino acid spacer to improve metabolic stability and 3-iodo-tyrosine to increase lipophilicity. This tracer precursor, termed "PSMA inhibitor for imaging and therapy" (PSMA I\&T), can be labelled with the usual radiometals, e.g. ${ }^{68} \mathrm{Ga},{ }^{111} \mathrm{In}$, and radiolanthanides. ${ }^{68} \mathrm{Ga}$-labelled and ${ }^{177} \mathrm{Lu}$-labelled radiopeptidomimetics have been studied in animals and patients [15] (Table 2).

A similar development/design has come from the German Cancer Research Center in Heidelberg [20]. The pharmacophore again is Glu-urea-Lys and the chelator is DOTA. The spacer is somewhat shorter, being composed of 2-naphthylalanine as a "super aromatic" amino acid and a derivative of aminomethyl-cyclohexane-carboxylic acid as the spacer's rigid part. Again, encouraging preclinical and clinical data have been generated [21] (Table 2). This ligand and others are commercially available.

\section{Systemic therapy using ${ }^{177}$ Lu PSMA ligands}

\section{Preliminary experience}

${ }^{177}$ Lu-PSMA ligand therapy in a cohort of ten patients [22] resulted in a PSA decline in seven patients (of whom five showed a decline of $>50 \%$ ); PSA progression was seen in three. 
Table 2 Published preliminary clinical experience with PSMA-targeted radioligands in CRPC

\begin{tabular}{|c|c|c|c|c|}
\hline Reference & $\begin{array}{l}\text { Compound and } \\
\text { regimen }\end{array}$ & Patients & Response & Toxicity \\
\hline [14] & $\begin{array}{l}{ }^{177} \mathrm{Lu}-\mathrm{J} 591 \\
\text { monoclonal } \\
\text { antibody, one } \\
\text { treatment with } 65 \\
\text { or } 75 \mathrm{mCi} / \mathrm{m}^{2}\end{array}$ & $\begin{array}{l}47 \text { who progressed } \\
\text { despite hormonal } \\
\text { therapy (55\% } \\
\text { also had prior } \\
\text { chemotherapy) }\end{array}$ & $\begin{array}{l}59.6 \% \text { any PSA decline, } 36.2 \% \\
\text { PSA decline } \geq 30 \%, 10.6 \% \\
\text { PSA decline } \geq 50 \% \text {. One patient } \\
\text { had partial radiographic response; eight } \\
\text { patients stable disease }\end{array}$ & $\begin{array}{l}55.3 \% \text { grade } 4 \text { thrombocytopenia } \\
\text { (29.8\% platelet transfusions); } \\
25.5 \% \text { grade } 4 \text { neutropenia } \\
\text { (one episode of febrile neutropenia) }\end{array}$ \\
\hline [15] & $\begin{array}{l}{ }^{177} \mathrm{Lu}-\mathrm{PSMA} \mathrm{I} \& \mathrm{~T}, \\
\text { one treatment } \\
\text { with } 5.7 \mathrm{GBq} \text { or } 8.0 \\
\text { GBq }\end{array}$ & $\begin{array}{l}\text { Two with CRPC } \\
\text { including multiple } \\
\text { bone and lymph } \\
\text { node metastases }\end{array}$ & $\begin{array}{l}\text { At } 3 \text { months, one patient had PSA } \\
\text { decrease from } 40.2 \mathrm{ng} / \mathrm{mL} \text { at baseline } \\
\text { to } 0.7 \mathrm{ng} / \mathrm{mL} \text {, partial remission of } \\
\text { numerous metastases on }{ }^{68} \mathrm{Ga} \text {-PSMA- } \\
\text { HBED-CC PET/CT, symptomatic pain } \\
\text { relief; results not reported for } 1 \text { patient }\end{array}$ & $\begin{array}{l}\text { No effect noted on blood counts, } \\
\text { renal function, other studied } \\
\text { biochemical analytes; "no } \\
\text { adverse or clinically detectable } \\
\text { pharmacological effect"; no side } \\
\text { effects, especially dry mouth, } \\
\text { observed }\end{array}$ \\
\hline [22] & $\begin{array}{l}{ }^{177} \mathrm{Lu}-\mathrm{DKFZ}-617, \text { one } \\
\text { treatment with } 4.1- \\
6.1 \mathrm{GBq} \text { (mean } 5.6 \\
\mathrm{Gbq})\end{array}$ & $\begin{array}{l}\text { Ten hormone-refractory } \\
\text { and/or chemorefractory } \\
\text { patients with distant } \\
\text { metastases and progressive } \\
\text { disease }\end{array}$ & $\begin{array}{l}\text { After } 8 \text { weeks, five patients had } \\
\text { PSA decline }>50 \%\end{array}$ & $\begin{array}{l}\text { No side effects immediately after } \\
\text { injection; one patient had grade } \\
3 \text { anaemia (causing fatigue and } \\
\text { leading to red blood cell infusion) } \\
\text { and grade } 2 \text { leucopenia } 7 \text { weeks } \\
\text { after treatment }\end{array}$ \\
\hline
\end{tabular}

$P C A$ prostate cancer, PSMA prostate-specific membrane antigen, PSMA I\&T PSMA inhibitor for imaging and therapy

None of the patients experienced any side effects immediately after injection. Relevant haematotoxicity (grade 3 or 4 ) occurred in just one patient 7 weeks after radioisotope administration. Six patients did not show any haematotoxicity at all throughout the 8 weeks after therapy. There was no relevant nephrotoxicity (grade 3 or 4). Indeed, to the best of our knowledge, no major toxicities have been reported so far in other cohorts. However, safety and tolerability have not been systematically assessed; such assessment should definitely be performed in every future clinical trial of PSMA-directed radionuclide therapy. Mild functional impairment of the salivary glands is not unlikely. External use of cooling pads in the region of the salivary glands may reduce salivary uptake of the ${ }^{177}$ Lu-PSMA ligand, and thus may be considered. The timing of this intervention has yet to be established, but from 30 min before until 4-6 h after injection may be one possibility. Salivary gland impairment should also be assessed systematically using a standardized questionnaire.

\section{Proposed protocol for ${ }^{177}$ Lu-PSMA ligand therapy}

Based on preliminary experience $[15,22]$, a practical recommendation for the use of ${ }^{177}$ Lu-PSMA ligand currently could be: patients with progressive metastatic CRPC would undergo therapy with $4-6 \mathrm{GBq}$ of ${ }^{177} \mathrm{Lu}$-PSMA per cycle, intravenously administered over $15 \mathrm{~min}$. Three cycles should generally suffice, although some patients might receive additional courses depending on individual responses and tolerance. A kidney protective effect of 2-phosphonomethyl-pentanedioic acid has been reported in mice, and this agent might ultimately be coadministered with the PSMA ligand [23].
Complete blood counts, parameters of renal function (serum creatinine, blood urea nitrogen), and liver function (albumin, bilirubin, enzymes), as well as tubular extraction rate measured by ${ }^{99 \mathrm{~m}} \mathrm{Tc}$-mercaptoacetyltriglycine scintigraphy, should be documented before and after therapy. Response to treatment should be assessed by ${ }^{68} \mathrm{Ga}$-PSMA PET combined with contrast-enhanced CT $8-10$ weeks after therapy. Additionally, cross-sectional radiological modalities, i.e. MRI, could be helpful in equivocal clinical situations. Biochemical response should be documented by monitoring PSA and alkaline phosphatase [15].

\section{Dosimetry of PSMA-targeted small molecules}

At the time of this report, only four studies have been published focusing on the radiation exposure of PSMA-targeted small molecules in humans: two studies on diagnostic imaging $\left({ }^{18} \mathrm{~F},{ }^{68} \mathrm{Ga}\right)[24,25]$, one on pretherapeutic dosimetry and subsequent therapy using ${ }^{124} \mathrm{I} /{ }^{131} \mathrm{I}$ as radionuclides [26], and one on pretherapeutic investigation with $200 \mathrm{MBq}$ of ${ }^{177} \mathrm{Lu}$ [27]. In these studies, besides increased uptake in the tumour tissue, kidneys, liver and spleen, uptake in the salivary glands and lacrimal glands was observed. The highest absorbed doses outside target tissues were seen in the kidneys and salivary glands. Bone marrow toxicity seems not to play a major role. The pretherapeutic study by Kabasakal et al. [27] has provided the only data published thus far for predicting therapy-related absorbed doses. However, this study had three drawbacks: 
1. The higher amount of unlabelled compound administered for therapy than for pretherapeutic imaging might have altered the pharmacokinetics and thus influenced the absorbed doses.

2. For quantification, the authors used planar imaging only. A recent review on dosimetry of ${ }^{177} \mathrm{Lu}$-DOTA compounds showed that the absorbed doses to the kidneys are systematically overestimated when using planar imaging [26].

3. No absorbed doses to the lacrimal glands were reported.

Therefore, more and reproducible data on dosimetry for treatment with radiolabelled PSMA-targeted molecules are urgently needed. The first efforts have been undertaken to standardize the calibration of scanners and dosimetry in the framework of the Metrology for Molecular Radiation Therapy (MetroMRT) project (http://projects.npl.co.uk/metromrt/) and Medical Internal Radiation Dose (MIRD) pamphlets 23 [28] and 26, the latter of which will shortly be published. However, a uniform method for performing dosimetry for ${ }^{177} \mathrm{Lu}$-labelled compounds has not yet been developed [29].

All dosimetry studies on therapeutic agents are at present hampered by the lack of adequate software for performing the necessary steps, i.e. image quantification, integration of the time-activity curve, and absorbed dose calculation. Nevertheless, basic standards and comparable protocols for dosimetry after therapy with PSMA-targeted compounds should be established. Only properly performed dosimetry studies, preferably in multicentre trials, can address the following open questions for the therapeutic application of PSMA-targeted small molecules:

(a) Are the absorbed doses to the target lesions sufficient for a therapeutic effect?

(b) What are the organs-at-risk and what are the absorbed doses that these organs could be expected to receive?

(c) Is fractionation of the treatment better than a single administration regarding safety and efficacy? If yes, what fractions and time intervals are optimal?

(d) Is one compound superior to the other regarding safety or efficacy?

\section{Conclusions}

${ }^{177}$ Lu-based PSMA-targeted therapy appears to be a promising treatment for advanced PCA. However, lessons should be learned from PRRT of neuroendocrine tumours, which was referred to as a "promising" tool for 15 years before the advent of evidence-based comparative studies. This experience strongly suggests that the communities involved with PSMA-targeted therapy, namely nuclear medicine, urology, radiochemistry, and medical physics, should capitalize without delay on the great opportunity to conduct well-designed prospective studies. Doing so should advance this modality from the proof-ofprinciple stage to the potential standard-of-care-stage. From our perspective, crucial components of this process are:

- Harmonization of therapy protocols

- Implementation of a patient selection algorithm into clinical routine

- Standardization of toxicity assessment

- Establishment of standardized dosimetry protocols to assess safety and efficacy

- Transfer of expertise in PSMA therapy throughout Europe

- Regulatory approval of ${ }^{177}$ Lu-PSMA-targeted compounds

Acknowledgments We acknowledge the contribution of Robert Marlowe in editing this paper.

\section{References}

1. Ferlay J, Soerjomataram I, Dikshit R, Eser S, Mathers C, Rebelo M, et al. Cancer incidence and mortality worldwide: sources, methods and major patterns in GLOBOCAN 2012. Int J Cancer. 2015;136: E359-86. doi:10.1002/ijc.29210.

2. Scher HI, Halabi S, Tannock I, Morris M, Sternberg CN, Carducci MA, et al. Design and end points of clinical trials for patients with progressive prostate cancer and castrate levels of testosterone: recommendations of the Prostate Cancer Clinical Trials Working Group. J Clin Oncol. 2008;26:1148-59. doi:10.1200/JCO.2007. 12.4487.

3. Petrylak DP, Tangen CM, Hussain MH, Lara Jr PN, Jones JA, Taplin ME, et al. Docetaxel and estramustine compared with mitoxantrone and prednisone for advanced refractory prostate cancer. N Engl J Med. 2004;351:1513-20. doi:10.1056/ NEJMoa041318.

4. Tannock IF, de Wit R, Berry WR, Horti J, Pluzanska A, Chi KN, et al. Docetaxel plus prednisone or mitoxantrone plus prednisone for advanced prostate cancer. N Engl J Med. 2004;351:1502-12. doi:10.1056/NEJMoa040720.

5. de Bono JS, Oudard S, Ozguroglu M, Hansen S, Machiels JP, Kocak I, et al. Prednisone plus cabazitaxel or mitoxantrone for metastatic castration-resistant prostate cancer progressing after docetaxel treatment: a randomised open-label trial. Lancet. 2010;376: 1147-54. doi:10.1016/S0140-6736(10)61389-X.

6. de Bono JS, Logothetis CJ, Molina A, Fizazi K, North S, Chu L, et al. Abiraterone and increased survival in metastatic prostate cancer. N Engl J Med. 2011;364:1995-2005. doi:10.1056/ NEJMoa1014618.

7. Ryan CJ, Smith MR, Fizazi K, Saad F, Mulders PF, Sternberg CN, et al. Abiraterone acetate plus prednisone versus placebo plus prednisone in chemotherapy-naive men with metastatic castrationresistant prostate cancer (COU-AA-302): final overall survival analysis of a randomised, double-blind, placebo-controlled phase 3 study. Lancet Oncol. 2015;16:152-60. doi:10.1016/S14702045(14)71205-7.

8. Scher HI, Fizazi K, Saad F, Taplin ME, Sternberg CN, Miller K, et al. Increased survival with enzalutamide in prostate cancer after chemotherapy. N Engl J Med. 2012;367:1187-97. doi:10.1056/ NEJMoa1207506.

9. Beer TM, Armstrong AJ, Rathkopf DE, Loriot Y, Sternberg CN, Higano CS, et al. Enzalutamide in metastatic prostate cancer before 
chemotherapy. N Engl J Med. 2014;371:424-33. doi:10.1056/ NEJMoa1405095.

10. Parker C, Nilsson S, Heinrich D, Helle SI, O'Sullivan JM, Fossa $\mathrm{SD}$, et al. Alpha emitter radium-223 and survival in metastatic prostate cancer. N Engl J Med. 2013;369:213-23. doi:10.1056/ NEJMoa1213755.

11. West TA, Kiely BE, Stockler MR. Estimating scenarios for survival time in men starting systemic therapies for castration-resistant prostate cancer: a systematic review of randomised trials. Eur J Cancer. 2014;50:1916-24. doi:10.1016/j.ejca.2014.04.004.

12. Jadvar H. PSMA PET in prostate cancer. J Nucl Med. 2015;56: 1131-2. doi:10.2967/jnumed.115.157339.

13. Ceci F, Uprimny C, Nilica B, Geraldo L, Kendler D, Kroiss A, et al. (68)Ga-PSMA PET/CT for restaging recurrent prostate cancer: which factors are associated with PET/CT detection rate? Eur J Nucl Med Mol Imaging. 2015;42:1284-94. doi:10.1007/s00259015-3078-6.

14. Tagawa ST, Milowsky MI, Morris M, Vallabhajosula S, Christos P, Akhtar NH, et al. Phase II study of lutetium-177-labeled anti-prostate-specific membrane antigen monoclonal antibody J591 for metastatic castration-resistant prostate cancer. Clin Cancer Res. 2013;19:5182-91. doi:10.1158/1078-0432.CCR-13-0231.

15. Weineisen M, Schottelius M, Simecek J, Baum RP, Yildiz A, Beykan S, et al. 68Ga- and 177Lu-labeled PSMA I\&T: optimization of a PSMA-targeted theranostic concept and first proof-ofconcept human studies. J Nucl Med. 2015;56:1169-76. doi:10. 2967/jnumed.115.158550.

16. Pandit-Taskar N, O'Donoghue JA, Durack JC, Lyashchenko SK, Cheal SM, Beylergil V, et al. A phase I/II study for analytic validation of 89Zr-J591 immunoPET as a molecular imaging agent for metastatic prostate cancer. Clin Cancer Res. 2015. doi:10.1158/ 1078-0432.CCR-15-0552.

17. Banerjee SR, Foss CA, Castanares M, Mease RC, Byun Y, Fox JJ, et al. Synthesis and evaluation of technetium-99m- and rheniumlabeled inhibitors of the prostate-specific membrane antigen (PSMA). J Med Chem. 2008;51:4504-17. doi:10.1021/jm800111u.

18. Eiber M, Maurer T, Souvatzoglou M, Beer AJ, Ruffani A, Haller B, et al. Evaluation of hybrid 68Ga-PSMA ligand PET/CT in 248 patients with biochemical recurrence after radical prostatectomy. $\mathrm{J}$ Nucl Med. 2015;56:668-74. doi:10.2967/jnumed.115.154153.

19. Maurer T, Weirich G, Schottelius M, Weineisen M, Frisch B, Okur $\mathrm{A}$, et al. Prostate-specific membrane antigen-radioguided surgery for metastatic lymph nodes in prostate cancer. Eur Urol. 2015;68: 530-4. doi:10.1016/j.eururo.2015.04.034.
20. Eder M, Schafer M, Bauder-Wust U, Hull WE, Wangler C, Mier W, et al. 68Ga-complex lipophilicity and the targeting property of a urea-based PSMA inhibitor for PET imaging. Bioconjug Chem. 2012;23:688-97. doi:10.1021/bc200279b.

21. Benesova M, Schafer M, Bauder-Wust U, Afshar-Oromieh A, Kratochwil C, Mier W, et al. Preclinical evaluation of a tailormade DOTA-conjugated PSMA inhibitor with optimized linker moiety for imaging and endoradiotherapy of prostate cancer. J Nucl Med. 2015;56:914-20. doi:10.2967/jnumed.114.147413.

22. Ahmadzadehfar H, Rahbar K, Kurpig S, Bogemann M, Claesener M, Eppard E, et al. Early side effects and first results of radioligand therapy with (177)Lu-DKFZ-617 PSMA of castrate-resistant metastatic prostate cancer: a two-centre study. EJNMMI Res. 2015;5: 114. doi:10.1186/s13550-015-0114-2.

23. Kratochwil C, Giesel FL, Leotta K, Eder M, Hoppe-Tich T, Youssoufian $\mathrm{H}$, et al. PMPA for nephroprotection in PSMAtargeted radionuclide therapy of prostate cancer. J Nucl Med. 2015;56:293-8. doi:10.2967/jnumed.114.147181.

24. Szabo Z, Mena E, Rowe SP, Plyku D, Nidal R, Eisenberger MA, et al. Initial evaluation of [18F]DCFPyL for prostate-specific membrane antigen (PSMA)-targeted PET imaging of prostate cancer. Mol Imaging Biol. 2015;17:565-74. doi:10.1007/s11307-0150850-8.

25. Herrmann K, Bluemel C, Weineisen M, Schottelius M, Wester HJ, Czernin J, et al. Biodistribution and radiation dosimetry for a probe targeting prostate-specific membrane antigen for imaging and therapy. J Nucl Med. 2015;56:855-61. doi:10.2967/jnumed.115. 156133.

26. Zechmann CM, Afshar-Oromieh A, Armor T, Stubbs JB, Mier W, Hadaschik B, et al. Radiation dosimetry and first therapy results with a (124)I/(131)I-labeled small molecule (MIP-1095) targeting PSMA for prostate cancer therapy. Eur J Nucl Med Mol Imaging. 2014;41:1280-92. doi:10.1007/s00259-014-2713-y.

27. Kabasakal L, AbuQbeitah M, Aygun A, Yeyin N, Ocak M, Demirci E, et al. Pre-therapeutic dosimetry of normal organs and tissues of Lu-PSMA-617 prostate-specific membrane antigen (PSMA) inhibitor in patients with castration-resistant prostate cancer. Eur J Nucl Med Mol Imaging. 2015. doi:10.1007/s00259-015-3125-3.

28. Dewaraja YK, Frey EC, Sgouros G, Brill AB, Roberson P, Zanzonico PB, et al. MIRD pamphlet no. 23: quantitative SPECT for patient-specific 3-dimensional dosimetry in internal radionuclide therapy. J Nucl Med. 2012;53:1310-25. doi:10.2967/ jnumed.111.100123.

29. Lassmann M, Eberlein U. Radiation dosimetry aspects of (177)Lu. Curr Radiopharm. 2015;8:139-44. 